\title{
Round pneumonia with Pseudomonas luteola and Escherichia vulneris bacteremia
}

Jacob Nichols MD, Weeraporn Srisung MD, and Shannon Yarbrough MD
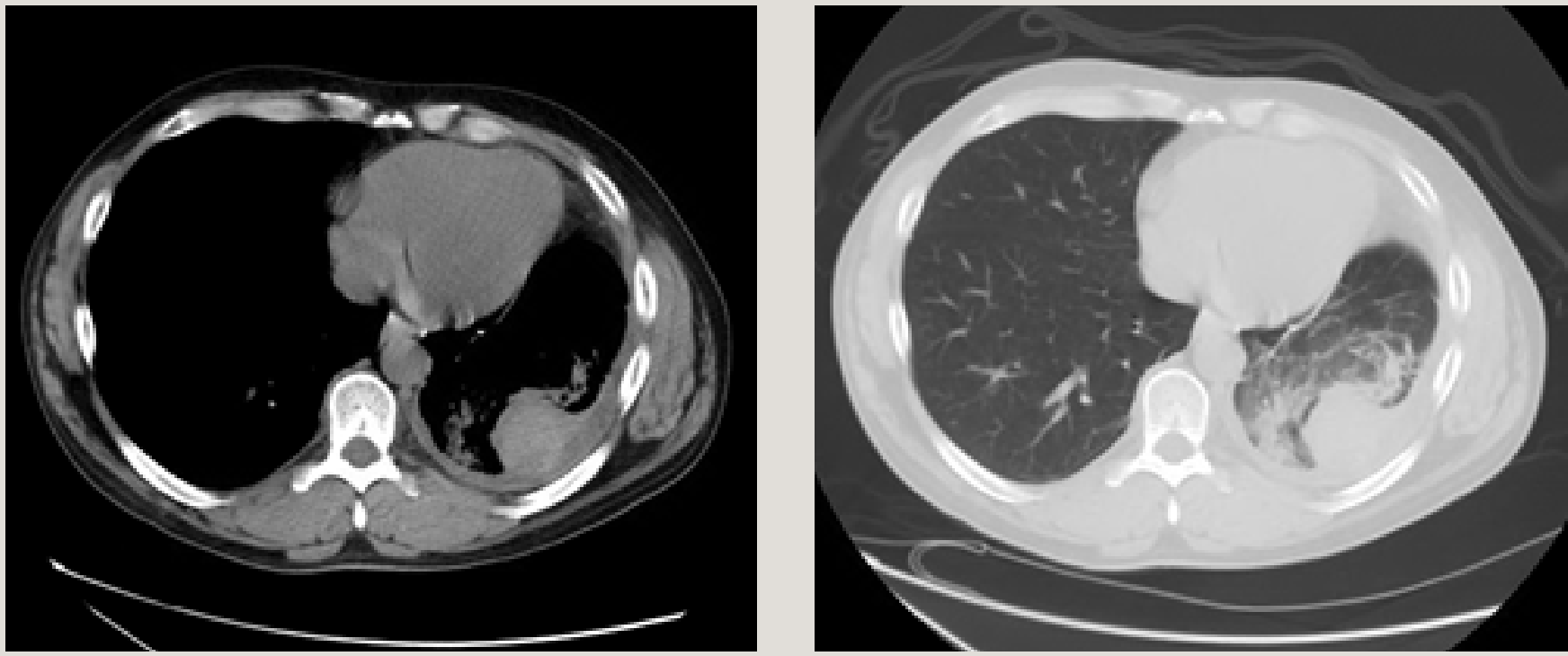

Figure 1

\section{CASE REPORT}

A 40-year-old man with a past medical history of alcohol abuse presented with altered mental status. The patient's wife reported that he had stopped drinking alcohol three days prior, and on the day of presentation had experienced two seizures and fever while at home. Physical examination at the time of admission revealed crackles over the left lower lung fields and a fluctuating mental status without focal neurological deficits or meningeal signs. Patient was febrile with a temperature of 101.4 F. Posteroanterior and lateral chest radiographs revealed a left sided pleural effusion with suspected pneumonia. After drawing blood cultures, he received ceftriaxone $1 \mathrm{~g}$ IV

Corresponding author: Jacob Nichols

Contact Information: Jacob.nichols@ttuhsc.edu

DOI: 10.12746/swrccc 2015.0312.153 and azithromycin 500mg IV in the emergency department. On the second day of admission, the patient's mental status had improved remarkably with treatment for suspected alcohol withdrawal with no further episodes of seizure, making the diagnosis of meningitis unlikely. He then reported he had experienced hemoptysis beginning five days prior to admission that lasted approximately three days. Later that day, blood cultures returned positive for two Gram negative rods, at which point piperacillin/tazobactam was started. It was decided to evaluate the lung infiltrates and pleural effusion by computed tomography (CT) of the chest which showed a $3.2 \mathrm{~cm} \times 6.0 \mathrm{~cm}$ rounded, slightly hyperdense lesion in the left lower lobe with an adjacent small pleural effusion. This lesion could represent a neoplasm, pneumonia, or hematoma (Figure 1). As the patient did not report any recent trauma in this region and had evidence of current infection, it was thought that this infiltrate was a round pneumonia. Blood culture speciation revealed Pseu- 
domonas luteola in two bottles and Escherichia vulneris in one bottle. Upon further questioning, it was discovered that the patient was a contractor, often requiring him to work in attics with exposure to insects, bird nests, and droppings without a mask. Thus, the diagnosis of round pneumonia with P. luteola and $E$. vulneris bacteremia was established, likely secondary to bacterial inhalation at work. Repeat blood cultures obtained after a single dose of ceftriaxone and azithromycin, but prior to piperacillin/tazobactam, showed clearance of the bacteremia. A PICC line was then placed and patient was discharged home to complete a 14 day course of IV ciprofloxacin, based on susceptibility testing of the initial blood cultures. The patient missed his initial follow up appointment and had to be rescheduled, at which point a repeat chest radiograph showed significant improvement in the effusion and infiltrates, 37 days after discharge.

\section{Discussion}

Round pneumonia is a condition most often seen in the pediatric population; however, it is occasionally seen in adults. ${ }^{1}$ On chest radiographs, these lesions typically appear as solitary pulmonary nodules, often first suspected to represent carcinoma of the lung which may require further evaluation by CT. In our case, it was the presence of the Gram negative rods in the blood culture that prompted further evaluation. Pseudomonas Iuteola (formerly Chryseomonas luteola) is a catalase positive, oxidase negative, motile Gram negative bacillus of the family Pseudomonaceae. ${ }^{2}$ While $P$. luteola infections are less common than those of $P$. aeruginosa, there have been case reports of the bacterium causing bacteremia, empyema, prosthetic valve endocarditis, post-surgical infections, and peritonitis. ${ }^{3-6}$ Although most often found in the soil and water sources, $P$. luteola has been isolated in animals, including turtles, tortoises and fish. ${ }^{7,8}$ Clinical isolates show susceptibility to third generation cephalosporins, mezlocillin, imipenem, aminoglycosides, and quinolones. The second pathogen, Escherichia vulneris, is a Gram negative, oxidase negative, indole negative, fermentative motile rod of the family Enterobacteriaceae that has been isolated from animals, drinking water, and humans. ${ }^{9}$ The bacterium is a known human pathogen found previously described causing bacteremia, as well as osteomyelitis, sepsis secondary to urinary tract infection, meningitis, and peritonitis. ${ }^{10-14}$ While $E$. coli is a cause pneumonia, a literature review did not reveal any previously reported cases of pneumonia involving E. vulneris. Animals known to carry the bacteria include wild birds and cockroaches. ${ }^{15,16}$ It displays a similar antibiotic sensitivity profile as E. coli and E. hermannii, generally with increased susceptibility. ${ }^{17}$ Although this bacterium grew in only one out of two bottles, it was considered a pathogen as the patient had significant occupational exposure to likely bacterial sources. Treatment duration of both bacteria is dependent on the site of infection. Treatment of round pneumonia is typically with antibiotics alone, requiring further evaluation if the lesion does not resolve on follow up imaging. Antibiotic therapy should be tailored to the suspected organism or based on culture and sensitivities, if available. Given our case, it should be noted that the presence of either P. luteola or $E$. vulneris should warrant consideration of a pulmonary source and a thorough occupational and exposure history should be obtained.

\begin{abstract}
Author affiliations : Jacob Nichols and Weeraporn Srisung are residents in Internal Medicine at Texas Tech University Health Sciences Center in Lubbock, TX. Shannon Yarbrough is a general internist in the Department of Internal Medicine at TTUHSC in Lubbock, TX.

Submitted: 9/6/2015

Accepted: $10 / 6 / 2015$

Reviewers: Eman Attaya MD

Conflict of Interest: None

Published electronically: 10/15/2015
\end{abstract}

\section{References}

1. Wagner AL, Szabunio M, Hazlett KS, Wagner SG. Radiologic Manifestations of round Pneumonia in Adults. Am J Roentgenology 1998; 170: 723-26.

2. Bayhan GI, Saliha S, Gonul T, Sengul O. Bacteremia Caused by Pseudomonas luteola in Pediatric Patients. Japanese $\mathrm{J}$ Inf Dis 2015; 68: 50-54.

$3 . \quad$ Yousefi F, Saeed S, Negin H. Empyema Caused by Pseudomonas luteola : A Case Report. Jundishapur J Micro 2014; 7: e10923.

4. Casalta JP et al. Prosthetic Valve Endocarditis Caused 
by Pseudomonas luteola. BMC Inf Dis 2005; 5: 82.

5. Kostman JR, Solomon F, Fekete T. Infections with Chryseomonas luteola (CDC Group Ve-1) and Flavimonas oryzihabitans (CDC Group Ve-2) in Neurosurgical Patients. Clinical Inf Dis 1991; 13: 233-36.

6. Su SY, Chao CM, Lai CC. Peritoneal Dialysis Peritonitis Caused by Pseudomonas luteola . Peritoneal Dialysis International: J Int Society Peritoneal Dialysis 2014; 34: 138-139. 7. Ianni FD, Pier LD, Clotilde SC, Igor P, Andrea S, Sandro C, Enrico P, Fausto Q, Simone T. Conjunctival Flora of Clinically Normal and Diseased Turtles and Tortoises. BMC Vet Research 2015; 11: 91.

8. Altinok I, Fikri B, Erol C, Sevki K. Disease of Rainbow Trout Caused by Pseudomonas luteola. Aquaculture 2007; 273: 393-97.

9. Kilani B, Lamia A, Hanène TB, Chadlia F, Taoufik BC. Escherichia vulneris as a Cause of Bacteremia in a Patient with Chronic Lymphocytic Leukemia. Int J Inf Dis 2008; 12: 110-11. 10. Spaulding AC, Rothman AL. Escherichia vulneris as a Cause of Intravenous Catheter-Related Bacteremia. Clinical Inf Dis 1996; 22: 728-29.

11. Levine WN, Goldberg MJ. Escherichia vulneris Osteomyelitis of the Tibia Caused by a Wooden Foreign Body. Ortho Reviews 1994; 23: 262-65.

12. Awsare SV, Lillo M. A Case Report of Escherichia vulneris Urosepsis. Clinical Inf Dis 1991; 13: 1247-248.

13. Mohanty S, Sharatp C, Benu D, Arti K, Bimalk D. Meningitis Due to Escherichia vulneris . Neurology India 2005; 53: 122 .

14. Senanayake SN, Jadeer A, Talaulikar GS, Roy J. First Reported Case of Dialysis-Related Peritonitis Due to Escherichia vulneris. J Clinical Micro 2006; 44: 4283-284.

15. Shobrak MY, Aly AE. Role of Wild Birds as Carriers of Multi-drug Resistant Escherichia Coli and Escherichia vulneris . Brazilian J Micro. 2014; 45: 1199-209.

16. Akbari S, Mohammad O, Saedeh SH, Sara H, Ghazaleh O, Mohammad HS. Aerobic Bacterial Community of American Cockroach Periplaneta Americana, a Step toward Finding Suitable Paratransgenesis Candidates. J Arthropod-Borne Dis 2015; 9: 35-48.

17. Stock I, Bernd W. Natural Antibiotic Susceptibility of Escherichia coli, Shigella, E. Vulneris, and E. hermannii Strains. Diagnostic Micro Inf Dis 1999; 33: 187-99. 